\title{
Adolescents in Primary Care with Sub-Threshold Depressed Mood Screened for Participation in a Depression Prevention Study: Co-Morbidity and Factors Associated with Depressive Symptoms
}

\author{
Benjamin W. Van Voorhees ${ }^{*}, 1,2,3$, Stephanie Melkonian ${ }^{1}$, Monika Marko ${ }^{1}$, Jennifer Humensky ${ }^{4}$ and \\ Joshua Fogel ${ }^{5}$ \\ ${ }^{I}$ Department of Medicine, The University of Chicago, Chicago, Illinois, USA \\ ${ }^{2}$ Department of Psychiatry, The University of Chicago, Chicago, Illinois, USA \\ ${ }^{3}$ Department of Pediatrics, The University of Chicago, Chicago, Illinois, USA \\ ${ }^{4}$ Center for Management of Complex Chronic Care, Edward Hines Jr. VA Hospital, Hines, IL, USA \\ ${ }^{5}$ Department of Economics, Brooklyn College of the City University of New York, Brooklyn, New York, USA
}

\begin{abstract}
Purpose: Adolescents in primary care with sub-threshold depression symptoms may be candidates for early intervention to prevent onset of full major depressive disorder. Little is known about this population.

Method: We screened consecutive adolescents (ages 14-21) in 13 primary care sites for presence of depression symptoms for "at least a few days" or "nearly every day" in the last two weeks for possible enrollment in a primary care/Internet-based depression prevention intervention (CATCH-IT). We report illness severity, prevalence of self-harm ideation, prevalence correlates (automatic negative thoughts, generalized self-efficacy, perceived social support from family and friends) and cooccurring symptoms of other mental disorders with depressed mood.

Results: Twenty-one percent $(\mathrm{N}=293)$ of those screened reported a core symptom of depressive disorder of which 83 enrolled in the study and were analyzed. The sample was $40 \%$ ethnic minority with a mean zip code household income of $\$ 40,249$ $(\mathrm{SD}=\$ 14,500)$. Reporting at least one symptom of another mental disorder was common for anxiety $(48 \%, \mathrm{~N}=40)$, substance abuse $(31 \%, \mathrm{~N}=15)$, conduct disorder $(71 \%, \mathrm{~N}=53)$, as were self-harm ideation $(16 \%, \mathrm{~N}=12)$ and reporting school impairment $(100 \%, \mathrm{~N}=83)$. Prevalence correlates for current depressive symptoms included low self-efficacy, automatic negative thoughts, perceived low peer acceptance, and school impairment.

Conclusions: Adolescents with sub-threshold depressed mood have frequent co-morbid symptoms that may need to be considered in developing prevention interventions. Early intervention targets to reduce depressed mood include pessimistic thinking, low self-efficacy, low peer acceptance, and school impairment.
\end{abstract}

Keywords: Depressive disorder, adolescents, attitudes, epidemiology, primary care, intervention.

\section{INTRODUCTION}

One-third of adolescents in primary care and $28 \%$ of those in high school settings report significant depressive symptoms. Less than one-third of these adolescents have symptoms meeting full criteria for major depressive disorder [1], but many of them will eventually progress to a diagnosis of major depressive disorder [2]. By age 24, one-quarter of adolescents will develop a depressive disorder with substantial adverse impact on both individuals and society with costs in excess of 100 billion dollars per year [3-6]. Regardless of the risk of progression, sub-threshold depressive symptoms at the level of minor depression with at least two core symptoms for at least 2 weeks result in considerable cost and impairment of social and academic function $[7,8]$.

*Address correspondence to this author at the Section of General Internal Medicine, Department of Medicine, The University of Chicago, 5841 South Maryland Blvd., Chicago, IL 60637, USA; Tel: 773-702-3835; Fax: 773-834-

2238; E-mail: bvanvoor@medicine.bsd.uchicago.edu
Primary care is a promising location to target adolescents with sub-threshold depression symptoms with early intervention programs to prevent onset of major depression [9]. Recent recommendations call for pediatricians and family physicians to play an enhanced role in screening for depressed mood and identifying and treating adolescents with depressive disorders [10-12]. Reports conducted in the United States, Europe, and by the World Health Organization have called for the development of early or preventive interventions targeting individuals with sub-threshold depressive disorder symptoms to reduce the burden of this disorder [13, 14]. Two primary care preventive interventions targeting adolescents with subthreshold depressive disorder symptoms have been developed $[9,15]$. Face-to-face cognitive behavioral and interpersonal psychotherapy interventions and supportive counseling in primary care have demonstrated benefit for adolescents with sub-threshold and mild levels of major depressive disorder $[16,17]$. 
Several studies have been conducted regarding screening for depressive disorder and other behavioral health problems in primary care $[1,12,18-20]$. These studies have validated paper and pen diagnostic tools, evaluated value of automatic feedback and assessed the general feasibility of the screening. However, to our knowledge, screening in view of identifying those vulnerable to future depressive disorder has not been undertaken. Screening is commonly used in primary care for other common somatic disorders such as cervical cancer and cardiovascular disease [21]. A screening approach for identification of those vulnerable to depressive disorder would enable investigators and public health officers to better understand the characteristics of the population that would likely be identified as candidates for prevention studies or interventions. Specifically, understanding the prevalence of core symptoms of depressed mood in the primary care population, co-morbid symptoms and disorders and prevalence correlates of depressed mood among those deemed appropriate candidates for prevention will facilitate further development of preventive interventions for this age group.

To our knowledge, there has been no description of a sample of adolescents at-risk for major depression in a primary care setting. Several papers do describe the cooccurrence and prevalence correlates of minor depression (one core symptom of depression plus 3 others, although some definitions vary) among adolescents in community settings $[22,23]$ and major depression in primary care [7]. Also, comorbid mental disorders are common with major depressive disorder [3] and may be common in an at-risk population for sub-threshold depression. There is some evidence that subthreshold depressed mood is more likely to co-occur with substance abuse, disruptive behaviors, and less likely to occur with anxiety disorders as compared to major depression [22]. A broader understanding of these patterns is essential to redesigning interventions and providing clinicians with a basis for counseling families.

Similarly, we do not know what factors are associated with sub-threshold depressed mood in an at-risk population of adolescents. Predictors of depressive disorder in general include cognitive/behavioral (e.g., negative interpretations and expectations, poor coping skills), interpersonal (e.g., social skills/problem solving deficits, lack of social support), and parental/ family factors (e.g., discord, high levels of criticism, lack of support, insecure attachment) [24, 25]. Similarly with regard to minor depression, negative discipline styles, family structure disruption (e.g., divorce), loss of caregiver, and neglect are known to correlate with minor depression among adolescents in a community sample [22]. We do not know if these factors or only certain ones (e.g., contextual factors such as family social support or more internal experiences such as automatic negative thoughts) are associated with sub-threshold depressed mood in primary care.

We have demonstrated that a primary care Internet-based preventive approach may reduce sub-threshold depression symptoms and risk for future depressive episodes [26]. We chose to focus on adolescents ages 14-21 because we believed that an intervention context would be very useful to focus on preventing depression during the years of high school through the possible transition to college. Providing a description of this primary care sample could provide those developing primary care interventions with a broader understanding of the phenomenon of sub-threshold depression in primary care. While this is not a representative epidemiologic survey of adolescent primary care patients, our goal was to complete a cross-sectional analysis of this prevention study screening sample. The focus of this study is to understand a targeted "atrisk" population, rather than the sampling frame of all adolescents with sub-threshold depressed mood. Consequently, this study does exclude some individuals deemed to be inappropriate for a prevention study. We measure prevalence of sub-threshold depressed mood depressive symptoms and co-morbid symptoms. We also determine the relationship of individual cognition and contextual factors such as family, peer, and school to these sub-threshold depressive symptoms.

\section{MATERIALS AND METHODS}

Study Design: We conducted a survey of adolescents enrolled in a randomized controlled trial of a primary care Internet-based depression prevention intervention. We recruited 13 primary care practices within five different health systems in four states (US Midwest and South). The target population of this study was adolescents identified through screening and willing to participate in an Internet-based public health model of depression prevention. The sampling frame was composed of adolescents identified by screening consecutive patients in primary care. Practices were selected to be similar to the range of US practice patterns (private practice, physician hospital organizations, public hospital, and federally qualified health centers) and on the basis of interest and geographic convenience. Recruitment occurred from February to November 2007 by screening of consecutive patients and with the use of fliers. We used a two-item screening questionnaire based on the core depression symptom items in the Patient Health Questionnaire Adolescent (PHQ-A) [1]. Adolescents reporting any core depressive symptoms (depressed mood, loss of pleasure or irritability) or depressive disorder lasting a minimum of a few days in the previous two weeks were considered positive screens. We contacted those with positive screens who granted permission (and their parent too if the participant was below age 18) by phone to conduct an eligibility assessment, which included the full PHQ-A assessment. The recruiting methods and study design have been described previously [26]. This study was approved by the University of Chicago Institutional Review Board, as well as those with responsibility for each site.

Adolescent Inclusion and Exclusion Criteria: Adolescents were between the ages of 14-21 years and experienced persistent sub-threshold depression $(>1$ core symptom of depression) at both the screening and eligibility assessment (12 weeks after initial screening). This study combined two protocols with slightly varying inclusion and exclusion criteria based on the degree to which the participant's own primary care physician was engaged with the study. Those adolescents who were enrolled in a community based trial protocol under the direct supervision of their primary care physician had less 
stringent criteria as the study worked with the culture and style of the primary care physician's environment. Both protocols excluded those meeting criteria (or undergoing active treatment) for major depression, expressing frequent suicidal ideation or intent, or meeting criteria for the following: bipolar, conduct, substance abuse and eating disorders. With regard to generalized anxiety and panic disorder, those who were enrolled in the community based trial protocol were not excluded while those enrolled in the university based protocol were excluded. Those who reported symptoms of conduct disorder, generalized anxiety disorder, or past substance abuse were not excluded in either group. Primary care physicians were granted some discretion in enrolling individuals with borderline major depression $(\mathrm{N}=3)$. In these cases, the local principal investigator, the study principal investigator, and the patient's physician agreed by consensus that the patient symptoms were sufficiently mild for the patient to enter the prevention study.

\section{Study Measures}

Prevalence of Mental Disorders: We determined the presence of major and minor depression (1 core symptom and 3 other symptoms nearly everyday for two weeks, as defined by the PHQ-A to indicate an episode not meeting full criteria for major depression (5 symptoms)) [1], sub-threshold depressed mood (presence of at least one core symptom at least a few days in last two weeks), dysthymia and other common disorders including generalized anxiety, panic disorder, alcohol abuse, substance abuse and eating disorders during phone assessments using the PHQ-A items. This instrument was developed as a self-report tool in primary care based on Diagnostic and Statistical Manual Version IV (DSMIV) criteria and has been validated by comparison to expert clinical assessments [1]. Additionally, we report proportions reporting symptoms of conduct disorder including lying, missing school (without excuse), stealing more than $\$ 20$ or bullying more than two times based on the Kiddie Schedule of Affective Disorders. This is a validated instrument for determining the presence of child mental disorder based on DSM-IV criteria [27]. Because the items are used in a selfreport instrument in this study, we only report positive responses as symptoms. For a dimensional measure of depressed mood, we report Center for Epidemiologic Studies Depression (CES-D, 20 item scale, score 0-60) scores and moodiness frequency ("How often have you felt moody in last 12 months?" with $0=$ never to $3=$ almost all the time). We also report PHQ-A scores (0-24) and PHQ-A-2 scores (core symptoms only, score 0-4). Responses to depression symptoms were assigned a numeric value (nearly every day $=$ 2 ; a few days $=1$; and not at all $=0$ ). The CES-D is a selfreport instrument intended for use in public and epidemiologic studies and has demonstrated reliability and validity in adolescent populations $[28,29]$.

Prevalence of Correlates of Depressed Symptoms: We collected baseline and follow-up assessments of individual vulnerability and protective factors including symptoms of other mental disorders, mood and affect regulation, cognition, and self-efficacy. Regarding negative cognition, we report the Automatic Negative Thoughts Questionnaire-Revised ( $0=$ not at all to $4=$ all the time; higher scores indicate more negative thoughts) [30]. This instrument was developed and has been validated as a measure of negative cognitions that are thought to either co-occur with/or predispose one to depressive episodes. This instrument can be administered relatively easily to adolescents as a self-report instrument and has demonstrated reliability and validity in this population $[4,30-$ 32], With regard to self-efficacy, we employed the scale developed by Pearlin for self-administration with a simple Likert scale ( $1=$ strongly agree to $4=$ strongly disagree; higher scores indicates lower self-efficacy) [31]. Items in each category were obtained from the National Longitudinal Study of Adolescent Health (ADD HEALTH), in which these items correlated with future risk of depressive disorder [33]. These items include: self-rated health ("In general how is your health?" with $1=$ excellent to $5=$ poor), self-rated intelligence ( $1=$ below to $6=$ above average), problem-solving skills (rational solving, $0=$ never to $3=$ nearly all the time) and selfefficacy with regard to affect regulation through behavior change ("I can change my depression by changing my behavior," with $1=$ strongly disagree to $5=$ strongly agree) [33]. These items were carefully chosen from a variety of sources for the ADD HEALTH study. The entire instrument underwent extensive pre-fielding assessments before fielding. We have demonstrated that these items predicted future depressive episodes in National Longitudinal Study of Adolescent Health data set [21,33].

Family, Peer and School Vulnerability and Protection: Adolescents reported the level of perceived family social support using the Perceived Social Support from Family (PSSfa) questionnaire. This instrument was developed to provide a simple self-report questionnaire to measure perceived social support from the adolescents' family and peers (separate instruments) and has demonstrated reliability and validity with adolescent populations [34]. Additionally, participants rated closeness to "Mom" and "Dad" and level of desire to leave home $(1=$ not at all to $5=$ quite a bit $)$ in items used in the ADD HEALTH questionnaire [33]. Adolescents rated perceived peer social support using the Perceived Social Support from Friends (PSS-fr) [34] and from the ADD HEALTH survey for items with regard to social acceptance ("I feel socially accepted"), and closeness to classmates ("I feel close to people at my school") ( $1=$ strongly agree to $5=$ strongly disagree). Additionally, we report level of school impairment related to depressed mood, based on items derived from the ADD HEALTH survey and interviews with emerging adults [35]. These items included reports of most recent grades in both Math and English ( $1=\mathrm{A}$ to $4=\mathrm{D}$ or lower) and agreement with the stem statement, "Feeling down or sad has affected my ability to do well in school" in the following ways (such as "concentrating in class" or "getting along with teachers," with $1=$ not at all to $4=a$ lot). The items with regard to impairment were combined into a scale with Cronbach alpha $=0.69$ and they were developed based on the responses of in-depth interviews with emerging adults suffering from depressive illness [35].

Statistical Analysis: We report percentages, proportions, means with standard deviations, and $95 \%$ confidence intervals where appropriate for the final sample of $\mathrm{N}=83$. We sought to 
explore the relationship between known prevalence correlates of depressive symptoms (CES-D score) in this primary care sample. We used linear regression with the continuous outcome measure of CES-D. Due to the small sample size, we were concerned about possibly over-adjusting with too many variables being included as possible independent variables. In model 1 , we conducted a linear regression analysis to evaluate the bivariate relationship between independent variables and the outcome. In model 2, we conducted the same analysis; however we adjusted for gender, age and ethnicity (dichotomized as white vs non-white). These analyses had a maximum of four independent variables included in the statistical model (e.g., A) Automatic Thoughts Questionnaire, gender, age and ethnicity; B) ADD HEALTH item on peer intelligence, gender, age and ethnicity etcetera). In model 3, we report results after adjusting only for those items in the same domain (e.g. cognition, family, peer and school). These domains were based upon previously published research identified from the ADD HEALTH study for depressed mood [36]. For example, for the domain of "family" the analyses only consisted of the four variables of perceived social support family scale, ADD HEALTH item of leave home, ADD HEALTH item of closeness to residential mother, and ADD HEALTH item of closeness to residential father. Those items found to be significant or with $p$-values $<0.10$ were advanced from model 3 to model 4 where 6 variables were entered together as part of the analysis. To adjust for the role of family structure on the outcome of depressed mood, we conducted two separate independent samples t-tests on family status as assessed by two variables: "parent married" vs "parent not married" and "living at home with married biological parents" vs not. We used STATA SE Version 10 for all the analyses.

\section{RESULTS}

Demographics: Twenty-one percent of those screened reported (negative screened reported fully in 5/13 clinics, so total number screened can only be estimated) a core symptom of depressive disorder $(\mathrm{n}=293)$ of which 116 were interviewed for eligibility ( $\mathrm{N}=46$ contacted but refused assessment, 131 could not be contacted). Of the 116 interviewed by phone, we excluded 13 who were ineligible [bipolar symptoms $(\mathrm{N}=2)$; major depression $(\mathrm{N}=2)$; self-harm intent $(\mathrm{N}=1)$; substance abuse $(\mathrm{N}=1)$; conduct disorder $(\mathrm{N}=1),<$ age $14(\mathrm{~N}=1)$, no depression symptoms $(\mathrm{N}=4)$; panic disorder $(\mathrm{N}=1)]$. Of the 103 eligible, 84 enrolled in the study $(82 \%$ enrollment rate, $29 \%$ of all those who screened positive), and 83 were included in this analysis (1 disenrolled). Table 1 shows the sample characteristics. Over half were female and the sample was ethnically diverse. The average age of the adolescents screened in primary care was slightly below 18 years, and slightly more than half reported their parents were married to one another. Approximately one-third had alternative living arrangements (i.e., not living at home with their married biological parents). Over half of the teens had completed at least 2 years of high school, while almost one-quarter were currently attending college. The mean household income for the zip codes of the participants according to the US Census 2000 was $\$ 40,249(\mathrm{SD}=\$ 14,500)[26]$.
Table 1. Demographics

\begin{tabular}{|c|c|c|}
\hline Characteristic & Proportion (Mean) & N (SD) \\
\hline \multicolumn{3}{|l|}{ Gender } \\
\hline Male & 43.82 & 36 \\
\hline Female & 56.18 & 47 \\
\hline \multicolumn{3}{|l|}{ Ethnicity } \\
\hline European American & 59.04 & 49 \\
\hline African American & 25.30 & 21 \\
\hline Hispanic & 4.82 & 4 \\
\hline Asian & 7.23 & 6 \\
\hline Native American & 0.00 & 0 \\
\hline Other & 3.61 & 3 \\
\hline Age & $(17.47)$ & $(2.04)$ \\
\hline \multicolumn{3}{|l|}{ Family Information } \\
\hline First born & 44.58 & 37 \\
\hline Non-first born & 50.60 & 42 \\
\hline Missing & 4.82 & 4 \\
\hline \multicolumn{3}{|l|}{ Parents Marital Status } \\
\hline Married & 53.01 & 44 \\
\hline Divorced & 19.28 & 16 \\
\hline Separated & 2.41 & 2 \\
\hline Never Married & 20.48 & 17 \\
\hline Missing & 4.82 & 4 \\
\hline \multicolumn{3}{|l|}{ Teen Living Situation } \\
\hline At home with Parents & 67.47 & 56 \\
\hline Alone & 2.41 & 2 \\
\hline With Friends or Roommates & 18.07 & 15 \\
\hline Other & 9.64 & 8 \\
\hline Missing & 2.41 & 2 \\
\hline Mean Income from Postal Code & $(\$ 40,249)$ & $(\$ 14,500)$ \\
\hline \multicolumn{3}{|l|}{ Father's Education } \\
\hline High School at least 2 years & 6.02 & 5 \\
\hline Finished high school & 30.12 & 25 \\
\hline College at least 2 years & 10.84 & 9 \\
\hline Finished college & 42.17 & 35 \\
\hline Missing & 10.84 & 9 \\
\hline \multicolumn{3}{|l|}{ Mother's Education } \\
\hline High School at least 2 years & 6.02 & 5 \\
\hline Finished high school & 25.30 & 21 \\
\hline College at least 2 years & 24.10 & 20 \\
\hline Finished college & 36.14 & 30 \\
\hline Missing & 8.43 & 7 \\
\hline \multicolumn{3}{|l|}{ Teen's Education } \\
\hline High School at least 2 years & 51.81 & 43 \\
\hline Finished high school & 12.05 & 10 \\
\hline College at least 2 years & 24.10 & 20 \\
\hline Finished college & 1.20 & 1 \\
\hline Missing & 10.84 & 9 \\
\hline
\end{tabular}


Table 2. Depressive Disorder and Symptoms

\begin{tabular}{|c|c|c|c|}
\hline & Proportion (Mean) & Number (SD) & $95 \% \mathrm{CI}$ \\
\hline \multicolumn{4}{|l|}{ Mood Measures } \\
\hline PHQ-A Score [mean scores] & $(7.36)$ & $(3.94)$ & $6.46,8.25$ \\
\hline \multicolumn{4}{|l|}{ Depressive Disorder } \\
\hline Major Depression & 0.04 & 3 & $0.01,0.11$ \\
\hline Minor Depression & 0.08 & 6 & $0.03,0.16$ \\
\hline Dysthymia & 0.01 & 1 & $0.00,0.08$ \\
\hline No depressive disorder & 0.80 & 66 & $0.70,0.87$ \\
\hline \multicolumn{4}{|l|}{ CESD Outcomes } \\
\hline Symptom free (CES-D $<14$ females, $<11$ males) & 0.19 & 16 & $0.12,0.29$ \\
\hline \multicolumn{4}{|l|}{ PHQ-A Self-Harm Risk } \\
\hline Self-harm thoughts last two weeks & 0.13 & 10 & $0.07,0.23$ \\
\hline Serious thoughts of suicide last month & 0.07 & 5 & $0.03,0.15$ \\
\hline Any self-harm thoughts & 0.16 & 12 & $0.09,0.26$ \\
\hline No thoughts of self harm & 0.75 & 62 & $0.64,0.83$ \\
\hline \multicolumn{4}{|l|}{ Depression Related School Impairment } \\
\hline Agrees with $>1$ school function Impairment by depressive disorder & 0.63 & 52 & $0.52,0.72$ \\
\hline
\end{tabular}

Exploration of Non-response: Out of those who did not participate in the initial phone assessment $(\mathrm{N}=167), 46$ answered the phone. We examined the factors contributing to not completing the follow-up survey call and we identified 4 key reasons why adolescents refused to enroll: "Too busy" $(\mathrm{N}=1)$, "Too young to participate" $(\mathrm{N}=10)$, "Declined/No reason given" $(\mathrm{N}=2)$, and "Not Interested" $(\mathrm{N}=23)$. With regard to depressed mood, we did a mean comparison of PHQ-A-2 scores between the enrolled participants $(\mathrm{N}=83)$ and those who did not enroll $(\mathrm{N}=101)$ and found no significant difference $(\mathrm{t}=0.65, \mathrm{p}>0.5)$. Additionally, we did a mean comparison of PHQ-A-2 scores between those who enrolled $(\mathrm{N}=83)$ and those who were eligible but chose not to enroll $(\mathrm{N}=20)$ and found no significant difference $(\mathrm{t}=0.37, \mathrm{p}>0.05)$.

Prevalence of Depressive and Co-morbid Disorders: Twenty-one percent of all those screened (derived from five clinics that retained all positive and negative screening forms) reported at least a few days of depressed mood in the last two weeks. Table 2 shows information for depressive and comorbid disorders. Slightly more than $10 \%$ had criteria for any type of depressive disorder. The mean CES-D score was in the moderately depressed range. Almost two-thirds reported at least some impairment in school and 16\% reported self-harm thoughts. Slightly less than one-third reported prior diagnosis and treatment for an emotional disorder and also almost onethird reported a family history of depression.

Co-Occurring Mental Disorders and Symptoms: Table 3 shows information for anxiety, substance, eating, and conduct disorders and symptoms. Nearly half reported at least some generalized anxiety symptoms, while almost one-fifth reported core substance abuse symptoms. While symptoms of eating disorders were common, no participants reached full criteria for either anorexia nervosa or bulimia nervosa. Conduct disorder symptoms were common with almost two-thirds reporting at least one symptom.

Factors Associated with Depressive Symptoms: Table $\mathbf{4}$ shows that in terms of cognition and self-efficacy, in the unadjusted model, all measures/items were significant for increased depressive symptoms except for depression selfefficacy. This included significance for more frequent negative thoughts, lower intelligence levels, less likely to look 
Table 3. Co-Occurring Mental Disorders and Symptoms

\begin{tabular}{|c|c|c|c|}
\hline \multicolumn{4}{|l|}{ Anxiety Disorders } \\
\hline Generalized AnxietyDisorder (full criteria) & 0.08 & 7 & $0.04,0.17$ \\
\hline Symptoms of GAD (Any core symptoms) & 0.45 & 37 & $0.34,0.55$ \\
\hline Panic disorder (full criteria) & 0.01 & 1 & $0.00,0.07$ \\
\hline Symptoms of Panic Disorder (Any core symptoms) & 0.13 & 11 & $0.07,0.22$ \\
\hline Any Anxiety Symptoms ( $>1$ core symptom either disorder) & 0.48 & 40 & $0.38,0.59$ \\
\hline \multicolumn{4}{|l|}{ Substance Abuse } \\
\hline \multicolumn{4}{|l|}{ Drug Abuse/Dependence } \\
\hline Drug Abuse/Dependence (full criteria) & 0.00 & 0 & N/A, N/A \\
\hline Symptoms of Drug Abuse (any core symptoms) & 0.13 & 11 & $0.07,0.22$ \\
\hline Any core symptoms of substance abuse & 0.18 & 15 & $0.11,0.28$ \\
\hline \multicolumn{4}{|l|}{$\begin{array}{l}\text { Eating Disorders } \\
\text { Bulimia Nervosa }\end{array}$} \\
\hline Bulimia Nervosa (full criteria) & 0.00 & 0 & N/A, N/A \\
\hline Symptoms of Bulimia Nervosa (any core symptoms) & 0.20 & 17 & $0.13,0.30$ \\
\hline Missed school $\geq 2$ times (without excuse) & 0.43 & 36 & $0.37,0.59$ \\
\hline Told $\geq 2$ lies & 0.14 & 12 & $0.09,0.26$ \\
\hline Fights $\geq 2$ times & 0.19 & 16 & $0.14,0.32$ \\
\hline Bullied another $\geq 2$ times & 0.13 & 11 & $0.08,0.25$ \\
\hline Stolen $>\$ 20$ on $\geq 2$ times & 0.06 & 5 & $0.03,0.15$ \\
\hline Any conduct disorder symptoms & 0.64 & 53 & $0.60,0.80$ \\
\hline
\end{tabular}

for multiple solutions to problems, lower self-rated health, and lower general self-efficacy. After adjustment for gender, ethnicity, and age (Model 2), intelligence levels no longer significantly predicted depressive symptoms. After adjusting for measures/items from the same category (Model 3), these same items were significant, except that less likely to look for multiple solutions to problems was no longer significant. In terms of family, no measures/items predicted depressive symptoms. In terms of peer, both lower perceived peer acceptance and closeness predicted increased depressive symptoms. This significance was maintained in Model 2 for both items and also in Model 3 for closeness while acceptance now only approached significance. In terms of school, increased perceived academic impairment predicted increased depressive symptoms in all 3 models. When all items with pvalues $<0.10$ from Model 3 were advanced to one model, more frequent negative thoughts, lower self-rated health, lower general self-efficacy, and greater school impairment remained significant predictors of depressive symptoms (Table 5).

\section{DISCUSSION}

Twenty-one percent of adolescents screened in primary care reported depressed mood persisting for at least two weeks. The vast majority exhibited sub-threshold depression 
Table 4. Linear Regression Models of Factors Associated with Depressive Symptoms (CES-D)

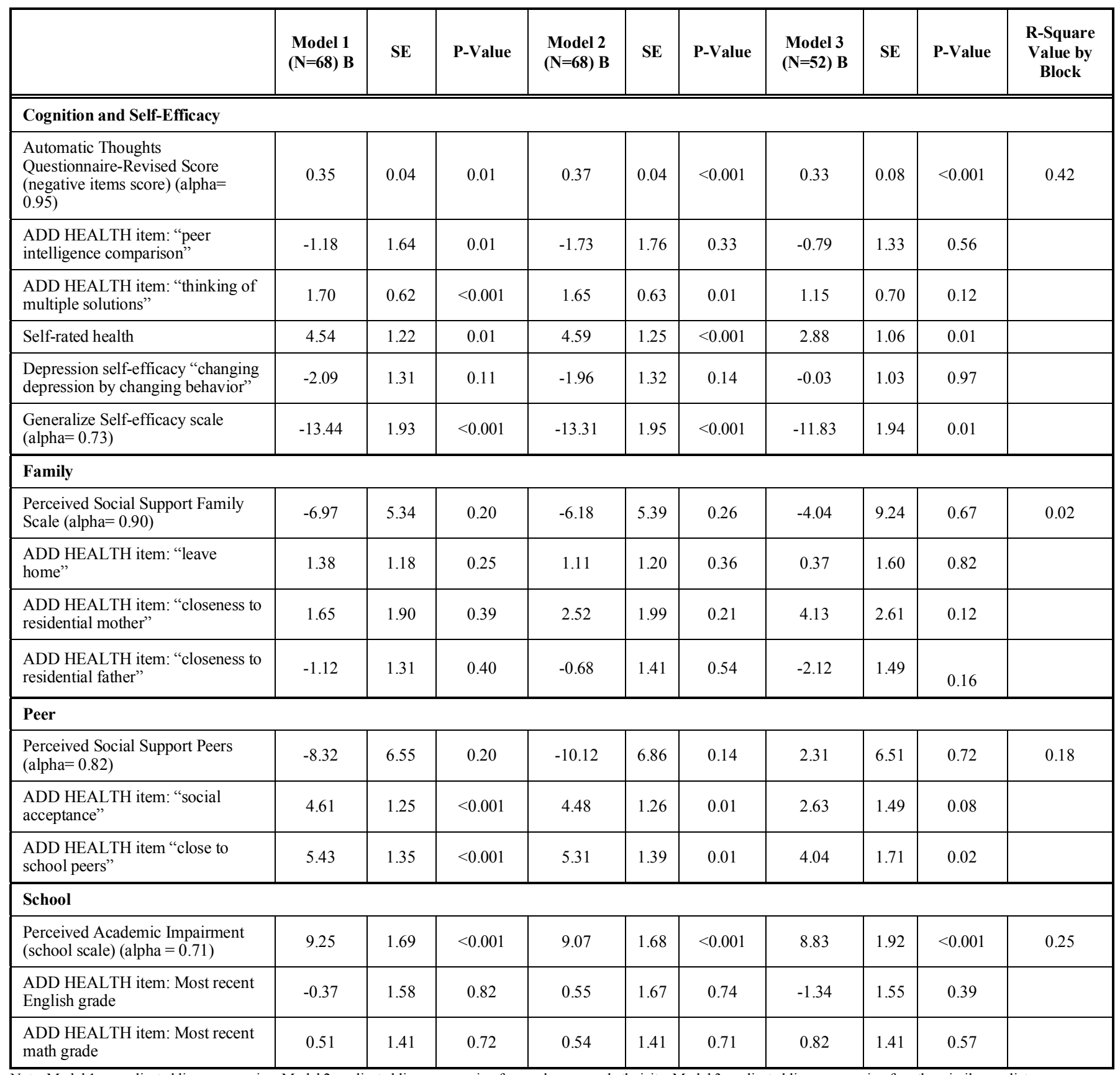

Note: Model 1 = unadjusted linear regression, Model 2 = adjusted linear regression for gender, age and ethnicity, Model 3 = adjusted linear regression for other similar predictors.

not achieving criteria for a depressive disorder. However, these individuals exhibited moderately depressed mood with most experiencing school impairment, co-occurring anxiety, or substance abuse and in a significant minority, self-harm ideation. Cognitive (automatic negative thoughts, low selfefficacy and self-rated health), peer (low acceptance/ closeness), and school (impairment) factor were associated with greater depressive symptoms.

The illness severity and complex co-morbid symptom patterns of sub-threshold depressed mood and factors that are associated with depressed mood in a primary care sample vulnerable to major depression has not been previously reported. The prevalence of depressed mood was lower than that observed in a United Kingdom primary care study $(21 \%$ vs $33 \%$ ) [20]. This lower prevalence of depressed mood may be the result of requiring at least a two week duration [1]. The findings of frequent co-occurrence of internalizing (e.g., anxiety), eating disorders, and "externalizing" symptoms (e.g., substance abuse and conduct disorder) are consistent with findings from the National Comorbidity Study for individuals with major and minor depressive disorder and suggest that those with sub-threshold symptoms in primary care exhibit a similar pattern [3]. The frequency of conduct disorder behaviors is consistent with other surveys [7] and suggests that 
Table 5. Linear Regression Models of Factors Associated with Depressive Symptoms (CES-D) (Combined Model of all those Items Significant or Approaching Significance $(p<0.10)$ in the Block regressions)

\begin{tabular}{|c|c|c|c|c|}
\hline & $B(N=64)$ & SE & P-Value & R-Square by Block \\
\hline \multicolumn{5}{|l|}{ Item } \\
\hline Self-rated Health & 2.11 & 0.91 & 0.02 & \\
\hline Generalize Self-efficacy Scale (alpha $=0.73$ ) & -4.73 & 2.13 & 0.03 & \\
\hline ADD HEALTH Item: "closeness to school peers" & -0.39 & 1.30 & 0.77 & \\
\hline Perceived Academic Impairment (school scale) (alpha $=0.71$ ) & 4.32 & 1.58 & 0.01 & \\
\hline
\end{tabular}

depressed mood and delinquent behaviors may be intertwined for many youth [37].

The finding that more frequent negative thoughts and low self-efficacy and perceived peer support, but not perceived family social support and connectedness, were associated with depressive symptoms, contrasts with earlier studies [24, 38] Lack of family connectedness is usually a strong predictor of future depressive episodes [33, 36]. Our findings suggest that perhaps current peer stressors or negative interpretations are more salient to these adolescents or conversely they have succeeded in eliciting more social support from their families by virtue of their depressed mood [39]. Our other findings are consistent with an extensive literature linking negative cognitions and peer related concerns to depressed mood [24]. Similarly, adolescents' reported levels of impairment of academic function were associated with level of depressive symptoms which is consistent with prior surveys of adolescents with major depressive disorder [20].

The strengths and limitations of the present study merit consideration. The sample was obtained from a well-selected sampling frame incorporating varying practice types, geographic locales, and two US regions using a systematic recruitment method (consecutive patients) and previously validated instruments. However, the large non-response from those who screened positive initially but who did not complete the phone for eligibility assessment should be considered. Neither the eligible potential participants nor those who could not be reached for assessment for eligibility differed from the sample in the severity of depressed mood. While this small sample demonstrated similar demographic characteristics of the adolescents enrolled to those of the US adolescent population (European American $61.2 \%$ vs $62.5 \%$ in the US population, and $38.8 \%$ minority vs $37.5 \%$ in the US population) with equal gender distribution, similarity in zip code income to national mean $(\$ 40,249$ vs $\$ 41,994$ (2000 census)) and proportion of adolescents living with their biological parents $(55.7 \%$ vs $52 \%$ for the entire US), its small size and the probable non-response bias can limit interpretations with regard to generalizability of findings [40]. Also, the ADD HEALTH item of "thinking of multiple solutions" may have changed from significance in Model 2 to not significant in Model 3 due to the smaller ratio of less than 10 individuals per variable. This exploratory analysis within a very small primary sample may serve to guide future larger primary care surveys.

\section{CONCLUSIONS}

In conclusion, the adolescents in primary care with subthreshold depression pose both a challenge and opportunity for physicians, investigators, and health planners. Currently recommended screening strategies for adolescents in primary care are likely to identify large numbers of adolescents with sub-threshold depressed mood. Physicians may wish to consider that one-fifth of their adolescent patients are experiencing persistent depressed mood for 2-4 weeks or more, and that many of their adolescent patients may have symptoms of other mental disorders too. It could be suggested that the identification of sub-threshold depressed mood should signify a concern to the clinician that the adolescent patient may have broader mental health and behavioral challenges crossing a range of disorders. Similarly, it may be helpful for the physician to recognize that the adolescent is experiencing these symptoms within an experiential framework of negative cognitions, lower peer support and generalized self-efficacy that may be amenable to counseling (either individual or family). For researchers and health planners, sub-threshold depressed mood is common and demonstrates predictors that are very amenable to current counseling and or emerging prevention strategies.

\section{ACKNOWLEDGEMENTS}

Supported by a NARSAD Young Investigator Award, Robert Wood Johnson Foundation Depression in Primary Care Value Grant and a career development award from the National Institutes of Mental Health (NIMH K-08 MH 072918-01A2).

\section{DISCLOSURES}

Benjamin W. Van Voorhees has served as a consultant to Prevail Health Solutions, Inc, Mevident, Inc, Social Kinetics, and the University of Hong Kong to develop Internet-based interventions.

\section{REFERENCES}

[1] Johnson JG, Harris ES, Spitzer RL, Williams JB. The patient health questionnaire for adolescents: validation of an instrument for the assessment of mental disorders among adolescent primary care patients. J Adolesc Health 2002; 30(3): 196-204. 
[2] Lewinsohn PM, Roberts RE, Seeley JR, Rohde P, Gotlib IH, Hops H. Adolescent psychopathology: II. Psychosocial risk factors for depression. J Abnorm Psychol 1994; 103(2): 302-15.

[3] Kessler RC, Walters EE. Epidemiology of DSM-III-R major depression and minor depression among adolescents and young adults in the National Comorbidity Survey. Depress Anxiety 1998; 7(1): 3-14.

[4] Cicchetti D, Toth SL. The development of depression in children and adolescents. Am Psychol 1998; 53(2): 221-41.

[5] Kessler RC, Avenevoli S, Ries Merikangas K. Mood disorders in children and adolescents: an epidemiologic perspective. Biol Psychiatry 2001; 49(12): 1002-14.

[6] Weissman MM, Wolk S, Goldstein RB, et al. Depressed adolescents grown up. JAMA 1999; 281(18): 1707-13.

[7] Asarnow JR, Jaycox LH, Duan N, et al. Depression and role impairment among adolescents in primary care clinics. J Adolesc Health 2005 ; 37(6): 477-83.

[8] Cuijpers P, Smit F, Oostenbrink J, de Graaf R, Ten Have M, Beekman A. Economic costs of minor depression: a population-based study. Acta Psychiatr Scand 2007; 115(3): 229-36.

[9] Van Voorhees BW, Ellis JM, Gollan JK, et al. Development and process evaluation of a primary care internet-based intervention to prevent depression in emerging adults. Prim Care Companion J Clin Psychiatry 2007; 9(5): 346-55.

[10] Richardson LP, Katzenellenbogen R. Childhood and adolescent depression: the role of primary care providers in diagnosis and treatment. Curr Probl Pediatr Adolesc Health Care 2005; 35(1): 6-24.

[11] Zuckerbrot RA, Cheung AH, Jensen PS, Stein RE, Laraque D; GLAD-PC Steering Group. Guidelines for Adolescent Depression in Primary Care (GLAD-PC): I. Identification, assessment, and initial management. Pediatrics 2007; 120(5): e1299-312.

[12] Zuckerbrot RA, Maxon L, Pagar D, Davies M, Fisher PW, Shaffer D. Adolescent depression screening in primary care: feasibility and acceptability. Pediatrics 2007; 119(1): 101-8.

[13] Bramesfeld A, Platt L, Schwartz FW. Possibilities for intervention in adolescents' and young adults' depression from a public health perspective. Health Policy 2006; 79(2-3): 121-31.

[14] Saxena S, Jané-Llopis E, Hosman C. Prevention of mental and behavioural disorders: implications for policy and practice. World Psychiatry 2006; 5(1): 5-14.

[15] Clarke GN, Hornbrook M, Lynch F. A randomized trial of a group cognitive intervention for preventing depression in adolescent offspring of depressed parents. Arch Gen Psychiatry 2001; 58(12): 1127-34.

[16] Cheung AH, Zuckerbrot RA, Jensen PS, et al. Guidelines for Adolescent Depression in Primary Care (GLAD-PC): II. Treatment and ongoing management. Pediatrics 2007; 120(5): e1313-26.

[17] Stein RE, Zitner LE, Jensen PS. Interventions for adolescent depression in primary care. Pediatrics 2006; 118(2): 669-82.

[18] Chisolm DJ, Klima J, Gardner W, Kelleher KJ. Adolescent behavioral risk screening and use of health services. Admin Policy Mental Health 2009; 36(6): 374-80.

[19] Ozer EM, Zahnd EG, Adams SH, et al. Are adolescents being screened for emotional distress in primary care? J Adolesc Health 2009; 44(6): 520-7.

[20] Yates P, Kramer T, Garralda E. Depressive symptoms amongst adolescent primary care attenders. Levels and associations. Soc Psychiatry Psychiatr Epidemiol 2004; 39(7): 588-94.

[21] Van Voorhees BW, Paunesku D, Gollan J, Kuwabara S, Reinecke M, Basu A. Predicting future risk of depressive episode in adolescents: the Chicago Adolescent Depression Risk Assessment (CADRA). Ann Fam Med 2008; 6(6): 503-11.

[22] González-Tejera G, Canino G, Ramírez R, et al. Examining minor and major depression in adolescents. J Child Psychol Psychiatry 2005; 46(8): 888-99.

[23] Sihvola E, Keski-Rahkonen A, Dick DM, et al. Minor depression in adolescence: Phenomenology and clinical correlates. J Affect Disord 2007; 97(1-3): 211-8.

[24] Hankin BL. Adolescent depression: description, causes, and interventions. Epilepsy Behav 2006; 8(1): 102-14.

[25] Reineck M, Simmons A. Vulnerability to depression among adolescents: implications for cogntive treatment. Cogn Behav Pract 2005; 12: 166-76.

[26] Van Voorhees BW, Fogel J, Reinecke MA, et al. Randomized clinical trial of an Internet-based depression prevention program for adolescents (Project CATCH-IT) in primary care: 12-week outcomes. J Develop Behav Pediatr 2009; 30(1): 23-37.

[27] Ambrosini PJ. Historical development and present status of the schedule for affective disorders and schizophrenia for school-age children (K-SADS). J Am Acad Child Adolesc Psychiatry 2000; 39(1): 49-58

[28] Radloff LS. The CES-D scale: a self-report depression scale for research in the general population. Appl Psychol Meas 1977; 1: 385401.

[29] Radloff LS. The use of the Center for Epidemiologic Studies Depression Scale in adolescents and young adults. J Youth Adolesc 1991; 20(2): 149-66.

[30] Kendall PC, Howard HB, Hays RC. Self-referent speech and psychopathology: the balance of positive and negative thinking. Cogn Ther Res 1989; 13: 583-98.

[31] Pearlin LI, Lieberman MA, Menaghan EG, Mullan JT. The stress process. J Health Soc Behav 1981; 22(4): 337-56.

[32] Kaufman NK, Rohde P, Seeley JR, Clarke GN, Stice E. Potential mediators of cognitive-behavioral therapy for adolescents with comorbid major depression and conduct disorder. J Consult Clin Psychol 2005; 73(1): 38-46.

[33] Van Voorhees BW, Paunesku D, Kuwabara SA, et al. Protective and vulnerability factors predicting new-onset depressive episode in a representative of U.S. adolescents. J Adolesc Health 2008; 42(6): 605-16.

[34] Procidano ME, Heller K. Measures of perceived social support from friends and from family: three validation studies. Am J Commun Psychol 1983; 11(1): 1-24.

[35] Kuwabara SA, Van Voorhees BW, Gollan JK, Alexander GC. A qualitative exploration of depression in emerging adulthood: disorder, development, and social context. Gen Hosp Psychiatry 2007; 29(4): 317-24.

[36] Paunesku D. Clusters of behaviors and beliefs predicting adolescent depression: implications for prevention. J Cogn Behav Psychother 2008; 8(2): 147-68.

[37] Beyers JM, Loeber R. Untangling developmental relations between depressed mood and delinquency in male adolescents. J Abnorm Child Psychol 2003; 31(3): 247-66.

[38] Compas BE, Hinden BR, Gerhardt CA. Adolescent development: pathways and processes of risk and resilience. Annu Rev Psychol 1995; 46: 265-93.

[39] Watson PJ, Andrews PW. Toward a revised evolutionary adaptationist analysis of depression: the social navigation hypothesis. J Affect Disord 2002; 72(1): 1-14.

[40] S.F.A.I.C. Fact Sheet of Demographics of Adolescents. USA: University of California 2003.

This is an open access article licensed under the terms of the Creative Commons Attribution Non-Commercial License (http://creativecommons.org/licenses/by-nc/ 3.0/) which permits unrestricted, non-commercial use, distribution and reproduction in any medium, provided the work is properly cited. 